\title{
ESTUDO EPIDEMIOLÓGICO LOCALIZADO DA FREQÜÊNCIA E FATORES DE RISCO PARA ENTEROPARASITOSES E SUA CORRELAÇÃO COM O ESTADO NUTRICIONAL DE CRIANÇAS EM IDADE PRÉ-ESCOLAR Parasitoses intestinais e desenvolvimento infantil
}

\author{
AN EPIDEMIOLOGICAL STUDY OF THE FREQUENCY AND FACTORS OF \\ RISK OF ENTEROPARASITOSES AND THEIR CORRELATION WITH THE \\ NUTRITIONAL STATE OF CHILDREN AT PRE-SCHOOL AGE \\ Intestinal parasitoses and infantile development
}

\author{
Helder Ferreira ${ }^{1}$, Eliane Raquel Peres Lala ${ }^{2}$, Marta Chagas Monteiro ${ }^{3}$, \\ Maria Lúcia Raimondo ${ }^{4}$ \\ 1 Autor para contato: Universidade Estadual de Maringá - UEM, Programa de \\ Pós-graduação em Análises Clínicas, Maringá, Paraná, Brasil; (44) 3263 0821; \\ e-mail: ferreira.helder@bol.com.br \\ 2 Universidade Estadual de Maringá - UEM, Programa de Pós-graduação em Ciências da \\ Saúde, Maringá, Paraná \\ 3 Universidade Estadual do Centro-Oeste - UNICENTRO, Departamento de Farmácia, \\ Guarapuava, Paraná. \\ 4 Universidade Estadual do Centro-Oeste - UNICENTRO, Departamento de Enfermagem, \\ Guarapuava, Paraná
}

Recebido para publicação em 09/10/2006

Aceito para publicação em 01/02/2007

\begin{abstract}
RESUMO
A parasitose intestinal é uma condição endêmica nos países em desenvolvimento, podendo apresentar manifestações diversas, desde as assintomáticas até a diarréia, perda protéica intestinal, desnutrição, anemia e dores abdominais. Tal problema afeta principalmente as populações de baixa renda, que vivem em condições precárias de saneamento básico e higiene, sendo as crianças as mais afetadas. Assim, visamos levantar o índice de parasitoses intestinais, o estado nutricional e o perfil de risco para endoparasitoses em crianças de uma creche municipal do município de Guarapuava-PR, correlacionando esses fatores. Foram analisadas 22 crianças com faixa etária entre 4 e 6 anos. O estado nutricional foi avaliado através de parâmetros antropométricos adotadas pela Organização Mundial da Saúde, usando a curva adotada pelo National Center of Health Statistics. Os dados socioeconômicos e os fatores de risco foram coletados por meio de formulário. As condições sanitárias das residências eram boas, 82\% recebiam água da rede pública, 86\% tinham coleta de lixo e 73\% possuíam
\end{abstract}


instalação sanitária interna. Após ir ao banheiro, somente 36\% das crianças lavavam as mãos. Apenas 9\% não tinham o hábito de andar descalças, enquanto $36 \%$ o faziam sempre e $55 \%$ somente às vezes. Do total das crianças estudadas, $50 \%$ relataram eliminação de vermes no passado e $45 \%$ queixavam-se de cólicas intestinais e diarréia. Dos exames cropológicos realizados 31,7\% apresentaram parasitas intestinais e destes aproximadamente $10 \%$ estavam com déficit nutricional. As crianças com déficit apresentavam menores condições socioeconômicas e de desenvolvimento antropómétrico. Constatou-se com a pesquisa uma estreita ligação entre condições de vida precária, parasitose intestinal e desenvolvimento inadequado.

Palavras-chave: parasitose, estado nutricional, fatores de risco

\begin{abstract}
Intestinal parasites are an endemic condition in developing countries, and they can present several manifestations that vary from the absence of symptoms to diarrhea, loss of intestinal proteins, malnutrition, anemia and abdominal pains. This problem mainly affects the populations of low income, that live in precarious conditions of basic sanitation and hygiene, the children being the most affected. In this study, our aim was to verify the rate of intestinal parasites, the nutritional state and the probability of risk of intestinal parasites in children of a municipal day nursery in Guarapuava-PR, establishing a correlation between these factors. Twenty-two children aged 4 to 6 were studied. Their nutritional state was evaluated through the anthropometric parameters adopted by the World Health Organization, by means of the curve used by the National Center for Health Statistics. The socioeconomic data and risk factors were collected through a questionnaire. The sanitary conditions of the houses were good, $82 \%$ received water from the public water supply, $86 \%$ had garbage collecting and $73 \%$ possessed internal sanitary installations. After going to the toilet, only $36 \%$ of the children washed their hands; only $9 \%$ had the habit of wearing shoes, while $36 \%$ always went barefoot and $55 \%$ occasionally did so. Also, 50\% of the children reported having excreted parasites in the past and 45\% complained of abdominal colic and diarrhea. 31.7\% of the exams presented intestinal parasites and $10 \%$ of these were of children with a nutritional deficit. These children with a nutritional deficit lived in worse socioeconomic and anthropometric conditions than the rest of the group. Thus, this research proved that there is a close connection among intestinal parasites, precarious life conditions and inadequate development.
\end{abstract}

Key words: parasites, nutritional state, risk factors

\section{Introdução}

A infecção parasitária, que afeta grande parte da população mundial, de acordo com Neves (2000), pode ser definida como a penetração e desenvolvimento, ou multiplicação, de um agente infeccioso no homem ou animal.

Quando se perguntam quais são as doenças mais 
comuns, poucas pessoas se lembram de incluir entre elas as parasitoses intestinais. No entanto, infecções causadas por parasitas intestinais são, sem dúvida, das doenças mais comuns e mais negligenciadas, afetando mais de 30\% da população mundial. (UNICEF, 1998).

Há cerca de 1,5 milhão de pessoas contaminadas com nematódeos. As trichiuras infectam um bilhão de pessoas, incluindo cerca de um terço de todas as crianças da África. Há mais de 1,3 bilhões de pessoas portadoras de ancilostomídeos no intestino, e 265 milhões de pessoas contaminadas por esquistossomos, colocando assim, a verminose como a terceira causa de infecções humanas mais comuns em todo o mundo. (UNICEF, 1998).

A criança tem-se mostrado o alvo da infecção parasitária e é nela que as repercussões da parasitose tornam-se mais significativas (Silva, 1997).

Segundo Behrman et al., (1997), as parasitoses infantis constituem uma causa importante de morbidade e mortalidade em todo o mundo. Estima-se que o ser humano seja infectado por mais de 100 tipos de parasitas, e estas infecções parasitárias sejam responsáveis por mais de um milhão de mortes por ano. Este mesmo autor enfatiza que em países industrializados, o número de casos de parasitoses em crianças tem aumentado muito nos últimos anos, revelando-se como um grave problema de saúde pública mundial.

Mesmo sendo a parasitose uma doença que faz parte do cotidiano de grande parte da população infantil mundial, trazendo-lhe danos secundários que podem afetar seu estado de saúde, trabalhos sobre o parasitismo intestinal em crianças ainda são escassos. Visando contribuir com maiores subsídios sobre o assunto é que a presente pesquisa foi desenvolvida, buscando conhecer o perfil de risco para verminose e o índice de parasitoses intestinais entre as crianças de uma creche da rede pública do município de Guarapuava, no ano de 2004, fazendo uma associação entre condições de vida, presença de parasitoses e dados antropométricos que traçam o desenvolvimento físico infantil.

A escolha do tema desta pesquisa tem como justificativa aspectos importantes, como a gravidade que assumem as parasitoses intestinais na infância, as condições socioeconômicas que são inerentes à questão e aos escassos estudos sobre a situação atual das infecções parasitárias em comunidades

\section{Materiais e métodos}

A população para esta pesquisa foi composta por alunos de uma creche da rede pública de ensino localizada na periferia do município de GuarapuavaPR, no ano de 2004. A amostragem foi não aleatória, formada por alunos com idade entre quatro e seis anos que freqüentam a creche. A creche era composta por 100 alunos. Dos 100 alunos, 50 são crianças abaixo de dois anos de idade. Para o estudo, foram selecionados alunos do jardim II, por se encontrarem na idade mais desejada para o estudo, que é entre quatro e seis anos. Dentre o total de 50 crianças selecionadas para o estudo, 28 (56\%) declinaram, pois não apresentaram o material para o exame parasitológico de fezes.

As crianças foram pesadas por meio de uma balança digital portátil e para a altura utilizou-se uma fita métrica fixada em uma parede. O estado nutricional foi avaliado através de parâmetros antropométricos (peso/altura) adotadas pela Organização Mundial da Saúde (OMS), usando à curva padrão adotada pelo National Center of Health Statistic (NCHS). Os exames parasitológicos de fezes foram realizados por um Laboratório de Guarapuava-PR.

A pesquisa foi realizada atendendo aos aspectos éticos definidos pela Resolução 196/96, do Conselho Nacional de Saúde (Brasil, 1996).

\section{Resultados}

No que concerne ao grau de escolaridade, observou-se que as mães possuem grau de escolaridade maior que os pais, sendo que $59 \%$ das mães possuem o ensino médio completo e enquanto apenas $23 \%$ dos pais possuem a mesma escolaridade. Em 32\% dos casos, não houve resposta quanto à ocupação e escolaridade do pai, pois tratava-se de mães solteiras ou divorciadas, que preferiram omitir essas informações, a figura paterna não era presente na relação.

As instalações sanitárias eram na maioria dos casos (73\%) localizadas no interior da residência. Em $82 \%$ das residências havia água encanada. Em 18\% dos casos a água era proveniente de poço e não houve relato de tratamento desta água antes do consumo, 
deixando assim, esse percentual em risco para a aquisição de verminoses.

O destino de esgoto, em $45 \%$ dos casos, era a rede pública, $41 \%$ fossa séptica e $14 \%$ fossa negra, ou seja, os dejetos são depositados à céu aberto podendo contaminar diretamente os indivíduos, ou indiretamente quando atinge lençóis e água como poços ou rios. O destino do lixo, 86\% dos casos era a coleta pública e $14 \%$ relataram que queimavam o lixo. Entre estas crianças, 36\% lavam as mãos sempre após ir ao banheiro, 64\% o fazem às vezes. Quanto a lavar as mãos antes de manipular alimentos, 73\% sempre lavam e 59\% lavam às vezes. Apenas 9\% disseram que não tem o hábito de andar descalço, enquanto $36 \%$ o fazem sempre e $55 \%$ somente às vezes.

Do total das crianças estudadas $50 \%$, relataram eliminação de vermes no passado e $45 \%$ queixava-se de cólicas intestinais e diarréia, 55\% já haviam realizado exames parasitológicos de fezes anteriormente. Destas, $9 \%$ apresentaram resultado negativo, e $27 \%$ tiveram diagnóstico de A. lumbricoides e 9\% G.lamblia. (Tabela 1).

Tabela 1 - Distribuição de freqüência dos sintomas relacionados à infecção parasitária e diagnósticos anteriores - Guarapuava, 2004.

\begin{tabular}{lcc}
\hline Variáveis & $\mathbf{n}$ & $\mathbf{\%}$ \\
\hline Cólicas intestinais e diarréia & 10 & 45 \\
$\quad$ Sim & 12 & 55 \\
$\quad$ Não & & \\
& & \\
Eliminou vermes anteriormente & & 50 \\
$\quad$ Sim & 11 & 50 \\
$\quad$ Não & 11 & \\
& & \\
Diagnósticos de exames & & \\
parasitológicos anteriores & & 27 \\
$\quad$ Ascaris lumbricoides & 6 & 9 \\
$\quad$ Giardia lamblia & 2 & 5 \\
$\quad$ A. lumbricoides e G. lamblia & 1 & 5 \\
$\quad$ Enterobious vermicularis & 1 & 5 \\
$\quad$ Negativo & 2 & 9 \\
$\quad$ Não fizeram & 10 & 45 \\
\hline
\end{tabular}

Amostra $=22$ crianças
A análise dos dados antropométricos das crianças participantes da pesquisa mostrou que $9,5 \%$ das crianças estavam com peso abaixo do preconizado para a idade. Destas com peso abaixo do preconizado para a idade, $5 \%$ apresentaram peso muito baixo para a idade e $5 \%$ apresentaram peso baixo para a idade. O restante das crianças (90\%), estão em peso normal para a idade.(Tabela 2).

Tabela 2 - Distribuição de freqüência da classificação do estado nutricional pelo indicador peso/idade Guarapuava, 2004.

\begin{tabular}{lcc}
\hline Variáveis & $\mathbf{n}$ & $\mathbf{\%}$ \\
\hline Peso muito baixo $(\mathrm{p} / \mathbf{i}<\mathbf{p - 0 , 1})$ & 1 & 5 \\
Peso baixo $(\mathrm{p}-0,1 \leq \mathrm{p} / \mathrm{i}<\mathrm{p}-3)$ & 1 & 5 \\
Peso normal $(\mathrm{p} / \mathrm{i} \geq \mathrm{p}-10)$ & 20 & 90 \\
\hline Amostra $=22$ crianças & & \\
\hline
\end{tabular}

Do total de amostras de fezes analisadas nesta pesquisa, 31,7\% apresentavam parasitas intestinais, sendo que as espécies de parasitas intestinais encontradas foram a Ascaris lumbricoides (4,5\%), Entamoeba coli $(13,6 \%)$ e Giárdia lamblia (13,6\%). Os 68,2\% restante não apresentaram nem uma alteração compatível com verminose. (Tabela 3).

Tabela 3 - Distribuição de freqüência dos resultados dos exames parasitológicos de fezes - Guarapuava, 2004.

\begin{tabular}{lcc}
\hline Variáveis & $\mathbf{n}$ & $\mathbf{\%}$ \\
\hline Ascaris lumbricoides & 1 & 4,5 \\
Entamoeba coli & 3 & 13,6 \\
Giardia lamblia & 3 & 13,6 \\
Negativo & 15 & 68,2 \\
\hline Amostra $=22$ crianças & &
\end{tabular}


O gráfico 1 nos mostra as crianças com diagnóstico positivo, $28 \%$ apresentaram déficit nutricional, sendo portadoras de A. lumbricoides e E. coli. O restante das crianças parasitadas (72\%) apresentaram um peso normal para a idade.

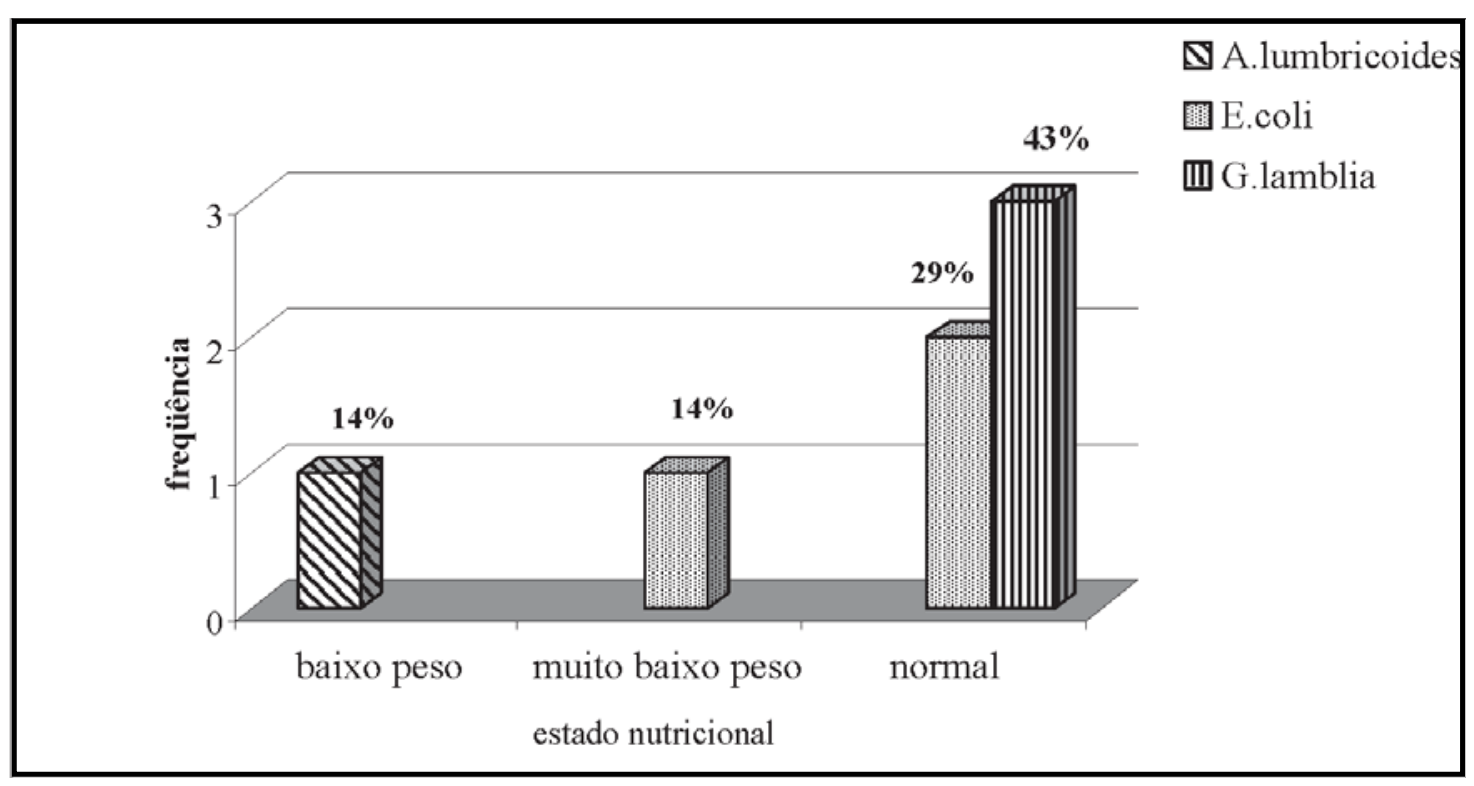

Gráfico 1 - Relação de parasitoses intestinais com estado nutricional - Guarapuava, 2004.

\section{Discussão}

A capacidade dos pais em cuidar dos seus filhos está intrinsecamente ligada à sua educação. Os pais envolvidos neste estudo possuem boa escolaridade, a maioria com ensino médio completo, favorecendo as condições de saúde de seus filhos, bem como o acesso ao trabalho e melhores condições de moradia.

Estudo realizado por Martins e colaboradores (2004), revelou, entre outros fatores, influencia significativa, da escolaridade materna e qualidade do ambiente, podendo supor que as mães com escolaridade tiveram mais acesso a informações sobre desenvolvimento infantil e que desta forma interagem melhor com seus filhos, respondem adequadamente às suas solicitações e podem prover melhores condições físicas e emocionais para o desenvolvimento de seu filho.

No início da década de 90, a adequação do cuidado infantil foi reconhecida como uma das três vertentes determinantes da prevenção da desnutrição e da promoção da saúde em geral, assim como a segurança alimentar, a salubridade do ambiente e o acesso a serviços de saúde (Carvalhes \& Benício,p.2,
2002). Segundo o mesmo autor, esse novo enfoque colocou no centro da atenção a capacidade dos pais de manejar e otimizar os diversos recursos necessários a um adequado cuidado, assim como o combate às deficiências nutricionais. Apesar disso, existem poucas evidências da relação entre capacidade dos pais de cuidar e estado nutricional na infância. Na realidade, o conceito de cuidado e seus possíveis indicadores e condicionantes ainda estão em desenvolvimento.

Em estudo realizado por Costa-Macedo (1999), a prevalência de parasitoses intestinais esteve significativamente associada às precárias condições socioeconômicas e ambientais.

Publicação do Ministério da Saúde mostrou que a infra-estrutura de saneamento básico no país ainda é desigual, estando concentrada nas áreas urbanas, nas regiões e nos estados com maior desenvolvimento econômico. Mesmo assim, ocorreu uma melhora acentuada do acesso da população aos serviços de saneamento básico em todo o país.

Dados do censo de 2000 identificou que a cobertura dos serviços de abastecimento público de água e da coleta de resíduos atendia três quartos da 
população brasileira. No entanto, a cobertura dos serviços de rede geral de esgotamento sanitário supria aproximadamente metade da população brasileira em 2000 (BRASIL, 2004).

É importante considerar que a fossa séptica ainda é uma modalidade de saneamento muito utilizada. Os estados da região Sul do país mostraram um percentual acentuado de uso deste tipo de esgotamento doméstico (BRASIL, 2004).

A contribuição para o declínio da mortalidade infantil decorrente de causas infecciosas e parasitárias é proveniente de ampliação do acesso ao saneamento básico, especialmente à água de boa qualidade (BRASIL, 2004). As formas de disseminação de parasitoses podem ser de veículos comuns como a água, os alimentos e o ar. A disseminação pode ocorrer de pessoa a pessoa, através do contato entre indivíduos infectados, com mãos contaminadas, vias aéreas ou através de seus dejetos que são eliminados a céu aberto (Neves, p11,2000).

As parasitoses intestinais podem apresentar manifestações diversas: desde assintomática até diarréia, perda protéica intestinal, desnutrição, anemia ferropriva e dores abdominais (Vitalle, 2003). Problemas este que facilmente levam a deficiência no desenvolvimento físico e intelectual.

As enteroparasitoses são um importante indicador das condições de saneamento em que vive uma dada população. Segundo a Unicef (1998), a população menor de cinco anos reflete bem o grau de contaminação de uma região, por tratar-se de indivíduos com pouca capacidade de deslocamento e maior vulnerabilidade, espelhando, assim, as condições sociais da comunidade onde habitam (Costa-Macedo, 1998). Segundo Behrman et al., (1997), os parasitas podem causar doenças por sua presença no organismo, por competição com o hospedeiro por nutrientes ou por resultado de tentativas do hospedeiro de destruir os invasores.

A diarréia crônica é um sintoma, freqüentemente, presente em crianças acometidas por parasitoses, podendo com isso levar a desnutrição pelas alterações na mucosa intestinal e infecção do trato gastrointestinal, não permitindo uma perfeita absorção levando a criança a apresentar alterações de desenvolvimento antropométrico e cognitivo.
Segundo Walker Smith (1989, p111), a íntima relação entre diarréia crônica e desnutrição em criança é bem conhecida, e esta "síndrome" é uma importante causa de morbidade e mortalidade. Existem relatos de déficits de atenção, mau desempenho em testes de inteligência, baixo rendimento escolar em matemática, baixo funcionamento motor, mudanças de comportamento e de humor, resultante da deficiência de ferro também em adolescentes. (Vitalle, 2003)

No Brasil, mais da metade de crianças préescolares e escolares encontram-se parasitadas (CostaMacedo, 1999). No ano de 2001, as doenças infecciosas e parasitárias ainda representaram a segunda causa de internações hospitalares na região Nordeste, e a terceira e a quarta causas de internações, respectivamente, nas regiões Sul e Sudeste do Brasil (BRASIL, 2004). Entre os parasitas mais encontrados está a Giárdia lamblia. Segundo Sogayar \& Guimarães (2000), creches são locais que apresenta condições favoráveis a transmissão de G.lamblia.

Cerca de 150 mil crianças morrem a cada ano no mundo, devido à obstrução intestinal e outras complicações abdominais causadas por parasitas intestinais. Para outros milhões de crianças, os vermes são uma importante causa de desnutrição, nanismo, anemia grave, diarréia, puberdade tardia, problemas de aprendizado e memória (UNICEF,1998).

Após análise dos dados das sete crianças parasitadas, observou-se uma relação entre o estado nutricional, a presença de parasitos intestinais, presença de fatores de risco para verminoses e a situação socioeconômica. Essas crianças apresentaram sintomas relacionados à infecção parasitária, todas relataram a ocorrência de cólica e diarréia, bem como a eliminação de vermes. Destas, apenas uma já havia realizado exame parasitológico de fezes. Quanto aos hábitos de higiene, apenas uma afirmou tomar as precauções básicas, como lavar as mãos antes de manipular alimentos, após ir ao banheiro e evitar andar descalço. Costa Junior e Zannon (1997), relata que nas últimas décadas, estudos sistemáticos tentaram delinear fatores sociais e determinantes do estado nutricional e do desenvolvimento biológico. De modo geral, observou-se que crianças procedentes de ambientes empobrecidos sofreriam uma dupla ameaça: estariam freqüentemente expostas a riscos de caráter ambiental e sofreriam, mais 
do que crianças de nível socioeconômico mais elevado, as conseqüências desses riscos. Além do fato de desnutrição e pobreza ocorrerem juntas, as crianças apresentariam menor resistência a infecções e maior número de seqüelas de doenças.

Concordamos com Costa Junior e Zannon (1997) o autor citado acima, pois as crianças estudadas que apresentaram diagnósticos positivos para parasitoses, estavam vivendo em condições socioeconômicas menos favorecida que as demais crianças. Analisando as condições de moradia destas crianças, observou-se a ausência de rede de esgoto nos domicílios, e em apenas três casos a água era proveniente da rede pública.

Em países em desenvolvimento, a desnutrição infantil é encontrada com freqüência em suas diversas formas, sendo um importante indicador das condições de saúde e da qualidade de vida de uma população (Marins,1995).

No Brasil, embora a desnutrição infantil tenha maior prevalência nas áreas do Nordeste, há indícios de microbolsões de desnutrição em áreas de baixa renda das grandes cidades da região Sudeste (Monteiro et al., 1988).

O acompanhamento da situação nutricional das crianças de um país constitui-se de um instrumento essencial para a aferição das condições de saúde da população infantil, sendo uma oportunidade ímpar para a obtenção de medidas objetivas da evolução das condições de vida da população em geral (Marins, 1995).

Segundo Marins (1995) a desnutrição ocorrida em crianças até cinco anos tem seus reflexos presentes em alterações na estatura e massa corporal, todavia, afeta também o desenvolvimento socioemocional e psicomotor, deixando seqüelas muitas vezes irreversíveis.

O Unicef, a OMS e o Banco Mundial selecionaram, crianças de pré-escola e em idade escolar para participarem de programas de controle de vermes, obtendo resultados espantosos. As crianças que receberam vermífugo não se queixaram mais de dores abdominais e nem dor de cabeça, como também a melhora do apetite. Estas crianças em nove semanas apresentaram melhor índice de crescimento e ganho de peso (depósito de gordura e ganho de massa muscular). Também se percebeu efeitos cognitivos e mentais da anemia sobre a criança, cujo desenvolvimento intelectual melhorou após o tratamento (UNICEF, 1998).

\section{Conclusão}

No presente estudo, observou-se um número relevante de pais ou responsáveis com ensino médio completo. A boa escolaridade dos pais destas crianças reflete nas melhores condições e qualidade de vida das famílias. Em um paralelo entre os achados, da pesquisa de dados e de exames laboratoriais, constatou-se que as crianças com diagnóstico de verminose positivo viviam em condições socioeconômicas menos favorecidas que as demais crianças. Mudanças neste quadro poderão ser conseguidas com o tratamento das crianças parasitadas e com mudanças nas condições ambientais nestas áreas de risco, para que haja melhoria de vida dos habitantes dessas comunidades, bem como uma intensificação nas ações de educação em saúde a fim de melhorar os hábitos de higiene de crianças e familiares, evitando a infecção cruzada de parasitas.

Todas as crianças que participaram e que mostraram resultados positivos para parasitoses foram encaminhadas para tratamento medicamentoso para eliminação dos parasitas e acompanhamento do desenvolvimento.

\section{REFERÊNCIAS}

1. BEHRMAN, R.E; KLIEGMAN, R.M.; ARVIN, A.M. Tratado de Pediatria. 15 ed. Rio de Janeiro: Guanabara Koogan, 1997.

2. BRASIL, Ministério da Saúde - Secretaria de Vigilância da Saúde. Saúde Brasil 2004 - Uma Análise da Situação de Saúde. Brasília 2004.

3. CARVALHAES M.A.; BENÍCIO, M.H.D’A. Capacidade materna de cuidar e desnutrição infantil Rev. Saúde Pública v.36, n.2, São Paulo, abr. 2002.

4. COSTA-MACEDO, L.M. Parasitismo pelo Ascaris lumbricoides em crianças menores de dois anos em comunidade aberta do Rio de Janeiro. Cadernos de Saúde Pública vol.15, p.173-178, 1999.

5. COSTA JUNIOR, A.L.; ZANNON, C.M.L.C. Desnutrição e desenvolvimento comportamental: questões metodológicas. Estudos de Psicologia v.2, n.2, Natal, jul./dez. 1997. 
6. COSTA-MACEDO, L.M. Enteroparasitoses em préescolares de comunidades favelizadas da cidade do Rio de Janeiro, Brasil. Cadernos de Saúde Pública vol. 4, p.851-855, 1998.

7. MARTINS M.F.D. Qualidade do ambiente e fatores associados: um estudo em crianças de Pelotas, Rio Grande do Sul, Brasil. Caderno de Saúde Pública, v.20, n.3, Rio de Janeiro, maio/jun. 2004.

8. MARINS, V.M.R.V. Perfil Antropométrico de Crianças de 0 a 5 anos do Município de Niterói, Rio de Janeiro. Caderno de Saúde Pública, v.11, n.2, Rio de Janeiro, abr./jun. 1995.

9. MONTEIRO, C.A.; CHIEFFI. P.P.; BENÍCIO M.H.A.; DIAS, R.M.S.; TORRES D.M.A.V.; MANGINI, A.C.S. Estudo das condições de saúde das crianças do Município de São Paulo (Brasil), 1984/1985. VII. Parasitoses intestinais. Revista de Saúde Pública vol. 22, p.8-15, 1988.

10. NEVES, D.P., Parasitologia Humana. 10 ed. São Paulo:
Atheneu, 2000.

11. SILVA N.R. Morbidity and mortality due to ascariasis: reestimation and sensivity analysis of global numbers at risk. Tropical Medicine International Health, vol.2, p.519-528, 1997.

12. SOGAYAR, M.I.T.L; GUIMARÃES, S. Giardia lamblia. In: NEVES, D.P., Parasitologia Humana. 10 ed. São Paulo: Atheneu, 2000.

13. UNICEF, Fundo das nações Unidas para a Infância. Situação Mundial da Infância. Brasília: Unicef, 1998.

14. VITALLE, M.S.S. Prevalência de anemia carencial ferropriva, parasitoses intestinais e estado nutricional em pacientes assistidos no Centro deAtendimento e Apoio ao Adolescente. Disponível em: <http://www.brazilpednews.org.br/marc2003/ anemia.pdf $>$. Acesso em: 02 julho 2003.

15. WALKER-SMITH, J.A. Diarréia e Desnutrição na Infância. Trad. I.M.Braun. Rio de Janeiro: Revinter,1989. Tradução de: Diarrhoea and Malnutrition in Childhood. 